\title{
A rare cause for peritonitis: pancreatic heterotopia
}

\author{
Wong Hoi She • Tan To Cheung • Kelvin Kwok Chai Ng
}

Accepted: 14 July 2010 /Published online: 31 July 2010

(C) The Author(s) 2010. This article is published with open access at Springerlink.com

\section{Dear Editor:}

Pancreatic heterotopia is defined as the presence of pancreatic tissue outside the pancreas without any anatomical or vascular connection with the pancreas itself. It can occur at any part of the gastrointestinal tract. Pancreatic heterotopia has been described as a silent anomaly since it is not clinically evident itself. Most of the time, it is diagnosed histologically. It is often presented as acute peritonitis. Occasionally it carries clinically detectable manifestations such as bleeding, biliary obstruction, and malignant transformation. It may lead to severe pancreatitis, a disease notorious for its morbidity and its $30 \%$ hospital mortality rate.

Since the first description of pancreatic heterotopia in 1,727 , there has been little information on its extreme presentation. We encountered a 62-year-old patient first diagnosed with acute pancreatitis and then peritonitis, but his real malady turned out to be pancreatic heterotopia. His case displayed the grave consequence of severe inflammation of ectopic pancreas located at the proximal jejunum.

The 62-year-old drinker having been suffering from epigastric pain and vomiting for some hours was admitted to our hospital; abdominal examination was carried out. Mild tenderness at the epigastrium without sign of peritonitis was detected. Bowel sound was active and succussion splash was positive. Blood tests showed an elevated white blood cell count $\left(28.4 \times 10^{9} / \mathrm{L}\right)$ and hyperamylasemia (505 U/L). Liver function, renal function, clotting profile, and blood gas were all normal. Chest and

W. H. She $\cdot$ T. T. Cheung $(\bowtie) \cdot$ K. K. C. Ng

Department of Surgery, The University of Hong Kong,

102 Pok Fu Lam Road,

Hong Kong, China

e-mail: tantocheung@hotmail.com abdominal X-ray displayed dilated gastric bubbles without free gas. The diagnosis was acute pancreatitis.

Eight hours later, the patient complained of aggravated epigastric pain. His condition deteriorated rapidly with development of high fever, tachycardia, and hypotension. Repeated physical examination showed apparent peritonitis in the abdomen. Chest X-ray was urgently repeated revealing free gas under the diaphragm.

Urgent laparotomy with midline incision was carried out. A liter of turbid peritoneal fluid was gathered. Surgical emphysema was found along the proximal small bowel mesentery, and a small perforation measuring $2 \mathrm{~mm}$ was discovered at the small bowel $10 \mathrm{~cm}$ from the duodenojejunal flexure. Inflammation on the mesenteric side of the proximal jejunum was located; whereas, there was no evidence of inflammation of the main pancreas. Segmental small bowel resection with end-to-end handsaw anastomosis was performed.

The patient was transferred to the intensive care unit after the operation because of severe septic shock. A high dose of infusion was administered to sustain his blood pressure. Broad-spectrum antibiotics piperacillin and tacarcillin which cover Gram-negative bacteria and anaerobic bacteria were dispensed. Postoperative blood tests indicated leukopenia, disseminated intravascular coagulopathy, and acute renal failure. A second-look laparotomy was carried out the following day but no abnormality was found; however, despite maximal support, the patient's conditions deteriorated and he died 2 days after the operation.

Histopathological examination of the specimen revealed the presence of pancreatic heterotopia at proximal jejunal wall, associated with acute pancreatitis and secondary bacterial infection resulted from perforation of the proximal jejunum. Gross pathological examination exposed a $2 \times 2 \times$ $1-\mathrm{cm}$ lobulated mural mass in the jejunum. Histology 
showed lobules of pancreatic acini and ductules as well as intervening smooth muscle fibers in a fibrous stroma. The mass was accompanied by suppurative inflammation, acute serositis, and focal transmural tissue necrosis. Gramnegative bacilli were also found. The overall picture was consistent with pancreatic heterotopia which was associated with acute inflammation, secondary bacterial infection, perforation, and acute peritonitis. Post-mortem examination was performed and no acute inflammation of the main pancreatic gland was found.

A congenital anomaly, heterotopic pancreas is pancreatic tissue existing aberrantly outside its normal anatomical location lacking both anatomic and vascular connections. It usually occurs at the upper gastrointestinal tract, the commonest sites being the duodenum $(27.5 \%)$, the stomach $(25.5 \%)$, and the jejunum (15.9\%). Unusual locations include the colon, the spleen, the liver, the biliary tract, the mesentery, and lymph nodes. Pancreatic heterotopia is a pathological diagnosis with an incidence estimated to be $0.5 \%$ to $15 \%$ of all autopsies with male predominance between the ages of 30 and 50 .

The pancreas is derived from the endoderm by means of fusion of the anterior and posterior pancreatic buds which arise from the caudal part of the foregut. The dorsal portion becomes the body and tail, and the ventral portion becomes the head of the pancreas. There are several hypotheses regarding the formation of heterotopic pancreas including abnormal attachment of pancreatic tissue to new locations at embryological state, abnormal differentiation of multipotent regional endoderm, and incomplete regression of the atrophic left ventral pancreatic bud.

Presentation of pancreatic heterotopia depends on the nature and location of a lesion. Patients of pancreatic heterotopia can be asymptomatic or they may have abdominal pain, intestinal obstruction, intussusception, jaundice, pancreatitis, cholecystitis, or benign tumors with malignant potential. In the worst case, they may die of the disease.

Preoperative diagnosis of pancreatic heterotopia is rarely possible regardless of improvements in diagnostic tools and techniques. The majority of patients only present the aforesaid symptoms with a raised amylase level. In a case of high suspicion, endoscopic ultrasound with fine needle aspiration can be used to help to confirm diagnosis of the disease. The image of most heterotopic pancreas produced by computed tomography is enhanced brightly similar to the image of orthotopic pancreas; whereas, reduced enhancement of image is a result of necrosis of heterotopic pancreas.

As imaging has its limitations, the aforementioned symptoms should warrant an exploratory laparoscopy or laparotomy. However, even with laparotomy, sometimes coming to a conclusive diagnosis may be difficult owing to the gross similarity of the lesion of pancreatic heterotopia to other types of tumor lesions, e.g., gastrointestinal stromal tumor, carcinoid, and even gastric carcinoma. Frozen section would be helpful to establish the diagnosis and to avoid unnecessary extensive operations. However, if a definite diagnosis still cannot be reached, in most cases segmental resection will be done so as to avoid reoperation, just like segmental resection for small bowel lesions, Whipple operation in the bile duct, wedge resection in the stomach, etc.

In pancreatic heterotopia, if pancreatitis occurs, it is virtually impossible to know without imaging, surgical, or pathological proof whether it is localized in the heterotopic tissue or involves the whole pancreas. The current case has clearly shown that inflammation can occur solely in ectopic pancreas without involvement of the main gland. This may pose great difficulty in the management of the situation because ectopic pancreas is normally very small so usually before signs and symptoms can be seen severe inflammation has already occurred.

In clinical management of pancreatitis, it is advisable to perform an imaging modality by either ultrasound or computer tomography scan to delineate the etiology of the disease within $72 \mathrm{~h}$ after admission for patients with stable conditions. Urgent imaging should be arranged if severe pancreatitis as classified by the Atlanta Criteria develops. In the management of complicated pancreatic heterotopia, more aggressive intervention should be carried out before irreversible systemic results arise. In differential diagnosis for peritonitis, pancreatic heterotopia should be considered. In the current case, the patient suffered not only extensive emphysematous change of the small bowel but also peritonitis caused by perforation of the small bowel.

Treatment option for pancreatic heterotopia would be radical operation if local excision is not possible. Resection of the portion bearing heterotopic pancreatic tissue is also advisable, and surgical exploration should be done for all symptomatic patients even without definite histological diagnosis.

Conflict of interest The authors declare that they have no conflict of interest.

Open Access This article is distributed under the terms of the Creative Commons Attribution Noncommercial License which permits any noncommercial use, distribution, and reproduction in any medium, provided the original author(s) and source are credited. 